\title{
Non-Darcy behavior of two-phase channel flow
}

\author{
Xianmin $\mathrm{Xu}^{1, *}$ and Xiaoping Wang ${ }^{2, *}$ \\ ${ }^{1}$ LSEC, Institute of Computational Mathematics and Scientific/Engineering Computing, NCMIS, AMSS, \\ Chinese Academy of Sciences, Beijing 100190, China \\ ${ }^{2}$ Department of Mathematics, Hong Kong University of Science and Technology, Clear Water Bay, Kowloon, Hong Kong, China
}

(Received 15 February 2014; revised manuscript received 17 June 2014; published 19 August 2014)

\begin{abstract}
We study the macroscopic behavior of two-phase flow in porous media from a phase-field model. A dissipation law is first derived from the phase-field model by homogenization. For simple channel geometry in pore scale, the scaling relation of the averaged dissipation rate with the velocity of the two-phase flow can be explicitly obtained from the model which then gives the force-velocity relation. It is shown that, for the homogeneous channel surface, Dacry's law is still valid with a significantly modified permeability including the contribution from the contact line slip. For the chemically patterned surfaces, the dissipation rate has a non-Darcy linear scaling with the velocity, which is related to a depinning force for the patterned surface. Our result offers a theoretical understanding on the prior observation of non-Darcy behavior for the multiphase flow in either simulations or experiments.
\end{abstract}

DOI: 10.1103/PhysRevE.90.023010

PACS number(s): 47.56.+r, 47.55.dr, 68.35.Ct

\section{INTRODUCTION}

The study of porous media flow is of critical importance in many applications such as the petroleum industry, soil sciences, fuel cells, painting filtration, etc. One-phase flow in porous media is well understood so far. Darcy's law, first derived through experiments in the 19th century [1], represents a linear relationship between the filtration velocity $\mathbf{v}$ and the pressure gradient: $\mathbf{v}=-K \nabla p$. Darcy's law can also be rigorously derived from the Navier-Stokes equations by homogenization techniques [2-4] or by the volume averaging method [5-7]. Nonlinear corrections to Darcy's law have been studied under various situations (see Refs. [8,9] and reference therein).

The modeling of two-phase flows in porous media has been mostly based on empirical approaches [10], with an assumed analogy with single-phase flow. In particular, with $\mathbf{v}_{i}$ being the superficial or seepage velocity vector of phase $i$ relative to the fixed pores, the two-phase form of Darcy's law is taken to be

$$
\mathbf{v}_{i}=-\frac{k_{r i}}{\eta_{i}} K\left(\nabla p_{i}-\rho_{i} \mathbf{g}\right), \quad \frac{\partial s_{i}}{\partial t}+\nabla \cdot\left(s_{i} \mathbf{v}_{i}\right)=0,
$$

where $k_{r i}$ represents the relative permeability of phase $i, i=$ 1,2 , and $p_{1}-p_{2}=p_{c}$ defines the capillary pressure. Both $k_{r i}$ and $p_{c}$ are assumed to be dependent only on the saturations $s_{i}\left(s_{1}+s_{2}=1\right)$, and the explicit functional relationships are assumed to be known from experiments.

Although the above model has been widely used in petroleum engineering, soil science, as well as many other fields, it has long been argued that the model might be invalidated either by theory, experiments, or both. Shortcomings of the model include (i) no account of interfacial force being taken in the momentum equation and (ii) quasistatic capillary pressure curves being used for a highly dynamic flow process. Due to the complexity of two-phase flow in porous media, most of the studies concern mainly the pure

*Corresponding authors: xmxu@1sec.cc.ac.cn and mawang@ust.hk imbibition or pure drainage process, where a macroscopic fluid interface moves across flow in porous media (see, for example, Ref. [11]). There are also many experimental or numerical studies on flow in porous media for a pore-network model. Among them, Avraam et al.did extensive experiments to show how the (conventional and generalized) relatively permeabilities depend on various regimes of flow mechanics, the capillary number, the flow ratio, the viscosity ratio, etc. [12-14]. Recently, Tallakstad et al.carried out some interesting experiments on steady two-phase flow in a representative porous-medium volume, where two phases are mixed together and both drainage and imbibition interplay $[15,16]$. They report some highly nontrivial behavior of the pressure jump with respect to capillary number.

In this paper, we study the macroscopic behavior of twophase immiscible flow in a porous medium by using a phasefield model with the generalized Navier boundary conditions developed in Refs. [23,24]. By using the homogenization technique, we first derive a generalized macroscopic dissipation law, $\mathcal{R}(\mathbf{x})=-\nabla P(\mathbf{x}) \cdot \mathbf{U}(\mathbf{x})$, which is valid for multiphase flows in porous media. We then use the dissipation law to study the effect of contact lines and roughness of pore surfaces on the macroscopic behavior of the two-phase flow by considering some simple pore geometries. For two-phase Poiseuille flow in a homogeneous channel, our calculations show that Darcy's law still holds with the permeability significantly reduced due to the dissipation associated with the contact line slip. The analysis is consistent with the Tallakstad experiments $[15,16]$. We then consider the case in which the channel surface is chemically patterned. It is shown that the total dissipation displays the scaling behavior $\mathcal{R}=F U \propto F_{0} U+$ $\lambda U^{2}$ which includes a non-Darcy term $F_{0} U$ that arises from the surface inhomogeneities. This non-Darcy term gives the forcevelocity relation $U \propto\left(F-F_{0}\right)$ for the chemically patterned surfaces.

We would like to remark that the simple pore geometry assumption ignores the interaction of different layers and the high-dimensional effect. However, our analysis provides some new theoretical understanding of Darcy and non-Darcy behavior in two-phase flow in porous media induced by the 
extra dissipations near moving contact lines. The dependence of the permeability and of the depinning force on the behavior of the moving contact lines is quantitatively shown. We expect that the force-dissipation relation and the analysis in this paper will be helpful in further studies of general porous-medium geometry.

The structure of the paper is as follows: In Sec. II, we carry out a homogenization analysis for a nondimensionalized twophase flow model in porous media. A macroscopic dissipation relation is derived. In Sec. III, we analyze in detail the case when the pore is a channel. Some general discussions are provided to show the difference of our results with standard Leverett models. The generalization to a higher-dimensional case is briefly discussed. In Sec. IV, numerical experiments are illustrated and we verify the analysis of the previous section. Finally, we give a few concluding remarks in Sec. V.

\section{MACROSCOPIC DISSIPATION FOR TWO-PHASE FLOW IN POROUS MEDIA}

In Ref. [23], the Cahn-Hilliard-Navier-Stokes (C-H-N$\mathrm{S})$ equations with the generalized Navier boundary condition (GNBC) are proposed to described the behavior of the moving contact line:

$$
\begin{aligned}
\frac{\partial \phi}{\partial t}+\mathbf{v} \cdot \nabla \phi & =M \Delta \mu \\
\rho\left[\frac{\partial \mathbf{v}}{\partial t}+(\mathbf{v} \cdot \nabla) \mathbf{v}\right] & =\mathbf{F}-\nabla p+\eta \Delta \mathbf{v}+\mu \nabla \phi \\
\nabla \cdot \mathbf{v} & =0 .
\end{aligned}
$$

The first equation is the Cahn-Hilliard equation considering the convection of the fluid. $\phi$ is the general phase-field function. $\mu=-K \Delta \phi-r\left(\phi-\phi^{3}\right)$ is the chemical potential with the interface thickness $\xi=\sqrt{K / r}$ and the fluid-fluid interface tension $\gamma=2 \sqrt{2} r \xi / 3$. $M$ is a phenomenological mobility coefficient.

The second equation is the Navier-Stokes equation with additional term $\mu \nabla \phi$, the capillary force exerted to the fluid by the interface. For simplicity, we assume that the two fluids have the equal constant density $\rho$ and viscosity $\eta$.

The boundary conditions for the phase field $\phi$, the normal velocity, and the chemical potential are given by

$$
\begin{aligned}
\frac{\partial \phi}{\partial t}+v_{\tau} \partial_{\tau} \phi & =-\Gamma L(\phi), \\
v_{n} & =0, \quad \partial_{n} \mu=0,
\end{aligned}
$$

with $\Gamma$ being a positive phenomenological parameter. $v_{n}$ and $v_{\tau}$ are normal and tangential velocity, respectively. The generalized Navier boundary condition is proposed to describe the moving contact lines:

$$
\beta v_{\tau}=-\eta \partial_{n} v_{\tau}+L(\phi) \partial_{\tau} \phi
$$

Here, $\beta$ is a slip coefficient and the slip length is given as $\tilde{l}_{s}=\eta / \beta . L(\phi)=K \partial_{n} \phi+\partial \gamma_{w f}(\phi) / \partial \phi$, where $\gamma_{w f}(\phi)=$ $-\frac{\gamma}{4} \cos \theta_{s}\left(3 \phi-\phi^{3}\right)$ is the solid-fluid interface energy density (up to a constant) and $\theta_{s}$ is the static contact angle. $L(\phi) \partial_{\tau} \phi$ represents the uncompensated Young stress.
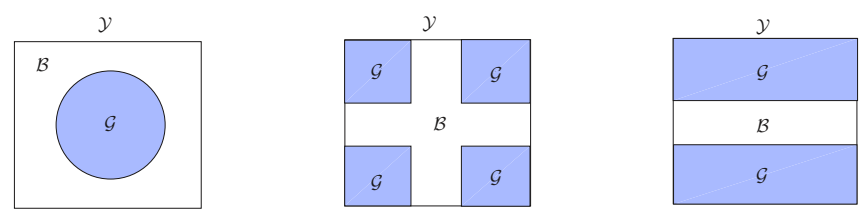

FIG. 1. (Color online) Examples of cell structures in periodic porous media.

\section{A. Nondimensionalization}

We consider two-phase flow in a porous medium. Define $\varepsilon=l / L$, the ratio between the pore length scale $l$ and the macroscopic scale $L$. We then scale the velocity by the characteristic velocity $v^{*}$, the length by $L$, the time by $L / v^{*}$, body force (density) $\mathbf{F}$ by $\eta v^{*} / l^{2}$, and the pressure by $L \eta v^{*} / l^{2}$. With five dimensionless parameters,

$$
\begin{aligned}
& \mathrm{L}_{\mathrm{d}}=\frac{3 M \gamma}{2 \sqrt{2} v^{*} l^{2}}, \quad \mathrm{R}_{\mathrm{e}}=\frac{\rho v^{*} l}{\eta}, \quad \mathrm{B}=\frac{3 \gamma}{2 \sqrt{2} \eta v^{*}}, \\
& \mathrm{~V}_{\mathrm{s}}=\frac{3 \gamma \Gamma l}{2 \sqrt{2} v^{*}}, \quad l_{s}=\frac{\tilde{l}_{s}}{l}, \quad \delta=\frac{\xi}{l},
\end{aligned}
$$

we have the following dimensionless Cahn-Hilliard-NavierStokes equations:

$$
\begin{aligned}
\frac{\partial \phi}{\partial t}+\mathbf{v} \cdot \nabla \phi & =\varepsilon^{2} L_{d} \Delta \mu, \\
\varepsilon \mathrm{R}_{e}\left[\frac{\partial \mathbf{v}}{\partial t}+(\mathbf{v} \cdot \nabla) \mathbf{v}\right] & =\mathbf{F}-\nabla p+\varepsilon^{2} \Delta \mathbf{v}+\varepsilon^{2} \mathrm{~B} \mu \nabla \phi, \\
\nabla \cdot \mathbf{v} & =0,
\end{aligned}
$$

where the chemical potential $\mu=-\varepsilon \delta \Delta \phi-\phi /(\varepsilon \delta)+$ $\phi^{3} /(\varepsilon \delta)$. The boundary conditions are

$$
\begin{aligned}
\varepsilon\left[\frac{\partial \phi}{\partial t}+v_{\tau} \partial_{\tau} \phi\right] & =-\mathrm{V}_{\mathrm{s}} \mathcal{L}(\phi), \\
l_{s}^{-1} v_{\tau} & =-\varepsilon \partial_{n} v_{\tau}+\varepsilon \mathrm{B} \mathcal{L}(\phi) \partial_{\tau} \phi, \\
\nabla \mu \cdot \mathbf{n} & =0, \quad \mathbf{v} \cdot \mathbf{n}=0,
\end{aligned}
$$

where $\quad \mathcal{L}(\phi)=\varepsilon \delta \nabla \phi \cdot \mathbf{n}+\partial \gamma_{w f}(\phi) / \partial \phi \quad$ and $\quad \gamma_{w f}(\phi)=$ $-\frac{\sqrt{2}}{6} \cos \theta_{s}\left(3 \phi-\phi^{3}\right)$ being the wall-fluid interface energy density function. $\mathbf{n}$ is the unit out normal on the boundary.

\section{B. Homogenization results}

We now derive the macroscopic behavior of the two-phase flow in porous media by the homogenization procedures. Let $\Omega \subset \mathbb{R}^{2}$ be a bounded domain which contains the porous medium under consideration. For simplicity, we assume the porous medium comprises a periodic array of small cells with size $\varepsilon \ll 1$. Denote the unit cell as $\mathcal{Y}$, which can be decomposed into two domains $\mathcal{B}$ and $\mathcal{G}$ (as shown in Fig. 1). Consider the porous domain defined by the fluid part $\Omega^{\varepsilon}=\Omega \cap \varepsilon\left(\cup_{\mathbf{z} \in \mathcal{A}}(\mathbf{z}+\mathcal{B})\right)$. Here, $\mathcal{A}$ is an infinite lattice in $\mathbb{R}^{2}$ such that $\cup_{\mathbf{z} \in \mathcal{A}}(\mathbf{z}+\mathcal{Y})=\mathbb{R}^{2}$ and $\left(\mathbf{z}_{1}+\mathcal{Y}\right) \cap\left(\mathbf{z}_{2}+\mathcal{Y}\right)=\varnothing$, for any $\mathbf{z}_{1}, \mathbf{z}_{2} \in \mathcal{A}$. 
We assume that the unknown velocity, pressure, and phasefield function have the following expansion:

$$
\begin{aligned}
& \mathbf{v}(\mathbf{x})=\mathbf{v}_{0}(\mathbf{x}, \mathbf{y})+\varepsilon \mathbf{v}_{1}(\mathbf{x}, \mathbf{y})+\varepsilon^{2} \mathbf{v}_{2}(\mathbf{x}, \mathbf{y})+\cdots, \\
& p(\mathbf{x})=p_{0}(\mathbf{x}, \mathbf{y})+\varepsilon p_{1}(\mathbf{x}, \mathbf{y})+\varepsilon^{2} p_{2}(\mathbf{x}, \mathbf{y})+\cdots, \\
& \phi(\mathbf{x})=\phi_{0}(\mathbf{x}, \mathbf{y})+\varepsilon \phi_{1}(\mathbf{x}, \mathbf{y})+\varepsilon^{2} \phi_{2}(\mathbf{x}, \mathbf{y})+\cdots,
\end{aligned}
$$

where $\mathbf{y}=\mathbf{x} / \varepsilon$. Assume $\mathbf{v}_{i}, p_{i}$, and $\phi_{i}$ to be all periodic in $\mathbf{y}$.

It is easy to see that the differential operators

$$
\nabla=\nabla_{x}+\varepsilon^{-1} \nabla_{y}, \quad \Delta=\Delta_{x}+2 \varepsilon^{-1}\left(\nabla_{x} \cdot \nabla_{y}\right)+\varepsilon^{-2} \Delta_{y},
$$

where $\nabla_{x}=\left(\partial_{x_{1}}, \partial_{x_{2}}\right)^{T}$ and $\nabla_{y}=\left(\partial_{y_{1}}, \partial_{y_{2}}\right)^{T}$, and $\Delta_{y}=$ $\partial_{y_{1} y_{1}}+\partial_{y_{2} y_{2}}$. Straightforward calculations show that the chemical potential $\mu$ can also be expanded as

$$
\mu(\mathbf{x})=\varepsilon^{-1} \mu_{0}(\mathbf{x}, \mathbf{y})+\mu_{1}(\mathbf{x}, \mathbf{y})+O(\varepsilon),
$$

with

$$
\begin{aligned}
& \mu_{0}=-\delta \Delta_{y} \phi_{0}-\phi_{0} / \delta+\phi_{0}^{3} / \delta, \\
& \mu_{1}=-\delta\left(2 \nabla_{x} \cdot \nabla_{y} \phi_{0}+\Delta_{y} \phi_{1}\right)-\phi_{1}\left(1-3 \phi_{0}^{2}\right) / \delta .
\end{aligned}
$$

Similarly, we have

$$
\mathcal{L}(\phi)=\mathcal{L}_{0}\left(\phi_{0}\right)+O(\varepsilon),
$$

with $\mathcal{L}_{0}\left(\phi_{0}\right)=\delta \nabla_{y} \phi_{0} \cdot \mathbf{n}+\partial \gamma_{w f}\left(\phi_{0}\right) / \partial \phi$. In addition, we introduce the fast time variable $s=t / \varepsilon$ and assume all quantities $f$ in the system to depend on $s$, i.e., $f=\phi_{i}, \mathbf{v}_{i}$ and is periodic in $s$ with period $T$. From the definition, we have $\frac{\partial f}{\partial t}=\frac{1}{\varepsilon} \frac{\partial f}{\partial s}$.

Substitute the expansions into above equations (7) and (8) (see details in Appendix A 1). The leading-order term of the second equation of Eqs. (7) gives

$$
\nabla_{y} p_{0}=0
$$

This implies that $p_{0}$ depends only on the slow parameter $\mathbf{x}$ and can therefore be taken to be the macroscopic pressure. To leading order, the velocity, phase function, and the chemical potential satisfy the following equations in the representative cell $\mathcal{Y}$ near $\mathbf{x}$

$$
\begin{aligned}
\mathrm{R}_{\mathrm{e}}\left[\frac{\partial \mathbf{v}_{0}}{\partial s}+\left(\mathbf{v}_{0} \cdot \nabla_{y}\right) \mathbf{v}_{0}\right] & =\mathbf{F}-\nabla_{x} p_{0}-\nabla_{y} p_{1}+\Delta_{y} \mathbf{v}_{0}+\mathrm{B} \mu_{0} \nabla_{y} \phi_{0} \quad \text { in } \mathcal{B}, \\
\nabla_{y} \cdot \mathbf{v}_{0} & =0 \quad \text { in } \mathcal{B}, \\
\frac{\partial \phi_{0}}{\partial s}+\mathbf{v}_{0} \cdot \nabla_{y} \phi_{0} & =\mathrm{L}_{d} \Delta_{y} \mu_{0} \quad \text { in } \mathcal{B}, \\
\mathbf{y} & \rightarrow \mathbf{v}_{0}, p_{1}, \phi_{0} ; \quad \text { periodic boundary condition on } \partial \mathcal{Y} \cap \partial \mathcal{B},
\end{aligned}
$$

with the boundary conditions on $\partial \mathcal{G} \cap \partial \mathcal{B}$,

$$
\begin{aligned}
\frac{\partial \phi_{0}}{\partial s}+v_{0, \tau} \nabla_{y} \phi_{0} \cdot \tau & =-\mathrm{V}_{\mathrm{s}} \mathcal{L}_{0}\left(\phi_{0}\right), \\
l_{s}^{-1} v_{0, \tau} & =-\left(\nabla_{y} v_{0, \tau} \cdot \mathbf{n}\right)+\mathrm{B} \mathcal{L}_{0}\left(\phi_{0}\right) \nabla_{y} \phi_{0} \cdot \tau, \\
\nabla_{y} \mu_{0} \cdot \mathbf{n} & =0, \quad \mathbf{v}_{0} \cdot \mathbf{n}=0,
\end{aligned}
$$

with $\mathcal{L}_{0}\left(\phi_{0}\right)=\delta \nabla_{y} \phi_{0} \cdot \mathbf{n}+\partial \gamma_{w f}\left(\phi_{0}\right) / \partial \phi$, and $\mu_{0}=-\delta \Delta_{y}$ $\phi_{0}-\phi_{0} / \delta+\phi_{0}^{3} / \delta$.

We define $P(\mathbf{x})=p_{0}(\mathbf{x})$ as the macroscopic pressure. Notice that since $\mathbf{v}_{0}$ is periodic in time $s$ with period $T$, the macroscopic velocity can be defined as

$$
\mathbf{U}(\mathbf{x})=\frac{1}{T|\mathcal{B}|} \int_{0}^{T} \int_{\mathcal{B}} \mathbf{v}_{0} d \mathbf{y} d s,
$$

with $|\mathcal{B}|$ being the volume of $\mathcal{B}$. In the case of one phase, low Reynolds-number flow, the macroscopic velocity can be solved from a simplified equation similar to the first equation in Eqs. (10), in terms of the pressure gradient. The usual one-phase Darcy law then follows, where the permeability is expressed in terms of the solution of a cell problem. However, such a derivation does not work for the two-phase system (10). We show, however, that a dissipation law is still valid.

\section{Dissipation function and Darcy's law}

We multiply by $\mathbf{v}_{0}$ the first of Eqs. (10) and integrate over $\mathcal{B}$. Direct computations give (see Appendix A 2)

$$
[\mathbf{F}-\nabla P(\mathbf{x})] \cdot \mathbf{U}(\mathbf{x})=\mathcal{R}(\mathbf{x}),
$$

where

$$
\begin{aligned}
\mathcal{R}(\mathbf{x})= & \frac{1}{T|\mathcal{B}|}\left[\int_{0}^{T} \int_{\mathcal{B}}\left|\nabla \mathbf{v}_{0}\right|^{2} d \mathbf{y} d s+\int_{0}^{T} \int_{\partial \mathcal{G}} \frac{v_{0, \tau}^{2}}{l_{s}} d \sigma_{y} d s\right. \\
& \left.+\frac{\mathrm{B}}{\mathrm{V}_{\mathrm{S}}} \int_{0}^{T} \int_{\partial \mathcal{G}} \dot{\phi}_{0}^{2} d \sigma_{y} d s+\mathrm{BL}_{\mathrm{d}} \int_{0}^{T} \int_{\mathcal{B}}\left|\nabla \mu_{0}\right|^{2} d \mathbf{y} d s\right]
\end{aligned}
$$

is the averaged dissipation rate over a cell [24].

The macroscopic behavior is characterized by Eq. (12). If we know how the averaged dissipation rate depends on $\mathbf{U}$, then an explicit relation between the force $\mathbf{F}-\nabla P$ and $\mathbf{U}$ may be obtained. In particular, if $\mathcal{R}$ scales quadratically with $\mathbf{U}$ (which is the case for the single-phase flow), then Darcy's law is recovered.

In the following, we consider only the simplest channel geometry in pore scale. It is shown that the Darcy law form still holds for smooth wall surfaces (with a permeability changed significantly by the effect of moving contact lines), but deviation from Darcy's law occurs when the surfaces are inhomogeneous. In the latter case, the dissipation function has precisely the form given by $\mathcal{R}=F U \propto F_{0} U+\lambda U^{2}$.

\section{TWO-PHASE CHANNEL FLOW}

We consider a channel of length $L_{x}$ and height $h$, i.e., $\mathcal{B}=$ $\left\{\left(y_{1}, y_{2}\right) \in\left(0, L_{x}\right) \times(0, h)\right\}$ and denote the upper and lower boundary of the channel $\partial \mathcal{G}=\left\{y_{2}=0, h ; 0<y_{1}<L_{x}\right\}$ (see Fig. 2). The two-phase flow in the channel is modeled by the 
C-H-N-S equations (10) with boundary conditions (11). For simplicity, we use $p, \phi, \mathbf{v}, \mu, \mathbf{F}, \mathcal{L}$, and $t$ instead of $p_{1}, \phi_{0}, \mathbf{v}_{0}, \mu_{0}$, $\mathbf{F}-\nabla_{x} p_{0}, \mathcal{L}_{0}$, and $s$, respectively. The equations are reduced to

$$
\begin{aligned}
\mathrm{R}_{\mathrm{e}}\left[\frac{\partial \mathbf{v}}{\partial t}+(\mathbf{v} \cdot \nabla) \mathbf{v}\right] & =\mathbf{F}-\nabla p+\Delta \mathbf{v}+\mathrm{B} \mu \nabla \phi \\
\nabla \cdot \mathbf{v} & =0 \\
\frac{\partial \phi}{\partial t}+\mathbf{v} \cdot \nabla \phi & =\mathrm{L}_{d} \Delta \mu, \\
\mathbf{y} & \rightarrow \mathbf{v}, p, \phi ; \quad \text { periodic boundary condition on }\left\{\mathrm{y}_{1}=0, \mathrm{~L}_{\mathrm{x}}\right\},
\end{aligned}
$$

with the boundary conditions on $\left\{y_{2}=0, h\right\}$,

$$
\begin{aligned}
\frac{\partial \phi}{\partial t}+v_{\tau} \nabla \phi \cdot \tau & =-\mathrm{V}_{\mathrm{s}} \mathcal{L}(\phi), \\
l_{s}^{-1} v_{\tau} & =-\left(\nabla v_{\tau} \cdot \mathbf{n}\right)+\mathrm{B} \mathcal{L}(\phi) \nabla \phi \cdot \tau, \\
\nabla \mu \cdot \mathbf{n} & =0, \quad \mathbf{v} \cdot \mathbf{n}=0,
\end{aligned}
$$

with $\quad \mu=-\delta \Delta \phi-\phi / \delta+\phi^{3} / \delta \quad$ and $\quad \mathcal{L}(\phi)=\delta \nabla \phi \cdot \mathbf{n}+$ $\partial \gamma_{w f}(\phi) / \partial \phi$. All the differential operators in the equations are now with respect to $\mathbf{y}$. We assume the external force $\mathbf{F}$ to be a constant vector (with respect to $\mathbf{y}$ ) along the $y_{1}$ direction.

\section{A. Homogeneous surfaces: Darcy's law in presence of contact line}

We now assume that the solid surface $\partial \mathcal{G}$ is chemically homogeneous. That is, $\theta_{s}$ is a constant along the boundary. With the constant external force $\mathbf{F}=\left(F_{1}, 0\right)$ in the $y_{1}$ direction, we can assume that the system (14) and (15) admits a travelingwave solution of the form

$$
\begin{aligned}
& \mathbf{v}(\mathbf{y}, t)=\mathbf{v}(\mathbf{y}-\mathbf{U} t), \\
& p(\mathbf{y}, t)=p(\mathbf{y}-\mathbf{U} t), \\
& \phi(\mathbf{y}, t)=\phi(\mathbf{y}-\mathbf{U} t),
\end{aligned}
$$

with a constant velocity $\mathbf{U}=(U, 0)$. The systems (7) and (8) are reduced to

$$
\begin{aligned}
\mathrm{R}_{\mathrm{e}}\{[(\mathbf{v}-\mathbf{U}) \cdot \nabla] \mathbf{v}\} & =\mathbf{F}-\nabla p+\Delta \mathbf{v}+\mathrm{B} \mu \nabla \phi, \quad \mathbf{y} \in \mathcal{B}, \\
(\mathbf{v}-\mathbf{U}) \cdot \nabla \phi & =\mathrm{L}_{d} \Delta \mu, \quad \mathbf{y} \in \mathcal{B},
\end{aligned}
$$

and

$$
\left(v_{\tau}-U\right) \partial_{\tau} \phi=-\mathrm{V}_{\mathrm{s}} \mathcal{L}(\phi), \quad \mathbf{y} \in \partial \mathcal{G} .
$$

We multiply by $\mathbf{U}$ the first equation of (16) and integrate over $\mathcal{B}$. We have, by direct computations (see Appendix A 3),

$$
F|\mathcal{B}|=\int_{\partial \mathcal{G}} l_{s}^{-1} v_{\tau} d \sigma_{y} .
$$

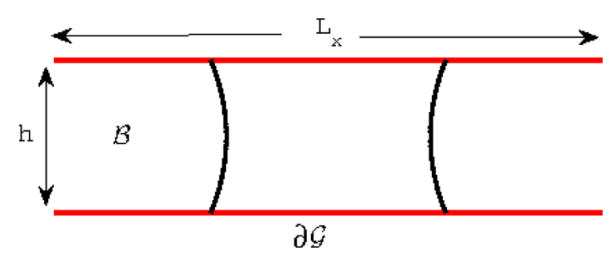

FIG. 2. (Color online) Two-phase flow in a channel with homogeneous upper and lower boundaries.
This implies that the external body force is balanced by boundary frictions.

The profile of the slip velocity near the moving contact lines on the solid boundary has been studied previously [25-27]. The velocity profile is found to have a universal behavior (see more discussion in Appendix B), i.e., $v_{\tau} / U \approx g\left[d\left(\mathbf{y}, \mathbf{y}_{i}\right) / l_{s}\right]$, where $d\left(\mathbf{y}, \mathbf{y}_{i}\right)$ is the distance from the $i$ th contact point $\mathbf{y}_{i}, g(\xi)$ is a universal function with a power-law slip region extending from a critical place about $r_{c}$ away from the contact line. In Ref. [25], it is found that $g(\xi)=1 /(1+\xi / a)$, with $a \approx 2.14$. The outer cutoff for the partial-slip region $\mathrm{R}$ is determined by the height $h$ of the channel. Beyond the partial-slip region, the slip velocity has a value given by $v_{s}=6 l_{s} U /\left(h+6 l_{s}\right)$ which can be derived from the Stokes equation for the single-phase Poiseuille flow with Navier slip boundary condition. For each contact point $\mathbf{y}_{i}$, it is shown that $\int_{\partial \mathcal{G} \cap\left\{\mathbf{y}: d\left(\mathbf{y}, \mathbf{y}_{i}\right)<\mathrm{R}\right\}} l_{s}^{-1} v_{\tau} d \sigma_{y} \approx \mathrm{A} U$, with some constant $\mathrm{A}$ independent of $U$. The value of $\mathrm{A}$ depends on the size of the power-law area. Thus, if we suppose that there are $n$ contact points on each side of $\partial \mathcal{G}$, then

$$
\int_{\partial \mathcal{G}} l_{s}^{-1} v_{\tau} d \sigma_{y} \approx\left(2 n \mathrm{~A}+\frac{12\left(L_{x}-2 n \mathrm{R}\right)}{h+6 l_{s}}\right) U,
$$

where $2 n$ is the number of contact points in the pore.

It follows from Eqs. (18) and (19) that

$$
U=\frac{h\left(h+6 l_{s}\right)}{12+2 n\left(\mathrm{~A} h+6 l_{s} \mathrm{~A}-12 \mathrm{R}\right) / L_{x}} F,
$$

and the permeability is given by

$$
k=\frac{h\left(h+6 l_{s}\right)}{12+2 n\left(\mathrm{~A}-\frac{12 \mathrm{R}}{h+6 l_{s}}\right) \frac{h+6 l_{s}}{L_{x}}} .
$$

Notice that for the one-phase flow, i.e., $n=0$, we recover the usual permeability expression for the slip boundary condition that $k_{0}=h\left(h+6 l_{s}\right) / 12$. In the presence of a contact line, however, the results in Refs. [25,27] show that $R$ is linearly proportional to $h$ and $\mathrm{A}$ behaves as $\ln \left(\mathrm{R} / l_{s}\right)$. Therefore, the expression $2 n\left[\mathrm{~A}-12 \mathrm{R} /\left(h+6 l_{s}\right)\right]\left(h+6 l_{s}\right) / L_{x}$ [in the denominator of Eq. (20)], which represents the contribution from the partial-slip dissipation near the contact line and which scales as $\left(h / L_{x}\right) \ln 10\left(h / l_{s}\right)$ for $h / l_{s}$ large, can significantly reduce the effective permeability. This is verified by our numerical experiments given in Table I.

From Eq. (21), the inverse of the permeability scales like

$$
k^{-1} \approx k_{0}^{-1}(1+b n),
$$

where $k_{0}$ is the standard permeability for one-phase porous media flow, $n$ is the total number of contact points in a 
TABLE I. Permeability: two-phase flow vs one-phase flow.

\begin{tabular}{lcccr}
\hline \hline & $l_{s}=1.6$ & $l_{s}=1.2$ & $l_{s}=0.8$ & $l_{s}=0.4$ \\
\hline$k$ & 122.10 & 113.01 & 103.54 & 93.57 \\
$k_{0}$ & 165.33 & 157.33 & 149.33 & 141.33 \\
\hline \hline
\end{tabular}

representative volume, and $b \propto \ln \left(h / l_{s}\right) h / L_{x}$, depending on both the geometry size and the slip length of the two-phase flows.

\section{B. Inhomogeneous surfaces: Non-Darcy behavior}

We now consider two-phase flow in a chemically patterned channel with the top and bottom surfaces patterned periodically (with small period, see Fig. 2) by two different materials with equal length but different contact angles $\theta_{a}$ and $\theta_{b}$. Without loss of generality, we assume $\theta_{a}>\theta_{b}$. We also assume that the motion of the two-phase flow is periodic in time with period $T$ and denote the time-averaged horizontal velocity as $U=\frac{1}{T|\mathcal{B}|} \int_{0}^{T} \int_{\mathcal{B}} v_{1} d \mathbf{y} d t$. It is reasonable to assume that $\frac{1}{T|\mathcal{B}|} \int_{0}^{T} \int_{\mathcal{B}} v_{2} d \mathbf{y} d t=0$ in the channel. Multiplying the first equation of (10) by $\mathbf{U}=(U, 0)$ and integrating over both space and time, we obtain

$$
\begin{aligned}
\mathbf{F} \cdot \mathbf{U}|\mathcal{B}|= & \operatorname{Re} \int_{\mathcal{B}} \partial_{t} \mathbf{v} \cdot \mathbf{U}+(\mathbf{v} \cdot \nabla) \mathbf{v} \cdot \mathbf{U} d \mathbf{y} \\
& -\int_{\mathcal{B}}(-\nabla p+\Delta \mathbf{v}+\mathrm{B} \mu \nabla \phi) \cdot \mathbf{U} d \mathbf{y} .
\end{aligned}
$$

By careful computations (see Appendix A 3), the equation is reduced to

$$
\begin{aligned}
F U L_{x} h T= & U \int_{0}^{T} \int_{\partial \mathcal{G}} l_{s}^{-1} v_{\tau} d \sigma_{y} d t \\
& -\frac{4 \sqrt{2}}{3} \mathrm{~B} U \int_{0}^{T} \int_{\partial \mathcal{G}} \cos \theta_{s}\left(y_{1}\right) \\
& \times \frac{\partial\left(3 \phi-\phi^{3}\right) / 4}{\partial y_{1}} d y_{1} d t
\end{aligned}
$$

For the first term on the right-hand side of Eq. (24), similar calculations as in homogeneous-boundary case give

$$
\int_{0}^{T} \int_{\partial \mathcal{G}} l_{s}^{-1} v_{\tau} d \sigma_{y} d t \approx\left(2 n \mathrm{~A}+\frac{12\left(L_{x}-2 n \mathrm{R}\right)}{h+6 l_{s}}\right) U T .
$$

Unlike in the homogeneous-surface case, the last term on the right-hand side of Eq. (24) no longer vanishes for the inhomogeneous surface, which is the origin of the non-Darcy effect. Assuming the solution is symmetric along the channel height, we then have

$$
\begin{aligned}
& \int_{0}^{T} \int_{\partial \mathcal{G}} \cos \theta_{s}\left(y_{1}\right) \frac{\partial\left(3 \phi-\phi^{3}\right) / 4}{\partial y_{1}} d y_{1} d t \\
& \quad=2 \int_{0}^{T} \int_{0}^{L_{x}} \cos \theta_{s}\left(y_{1}\right) \frac{\partial\left(3 \phi-\phi^{3}\right) / 4}{\partial y_{1}} d y_{1} d t .
\end{aligned}
$$

We estimate the above integral in the sharp-interface limit of the phase-field function. When the interface thickness $\delta$ goes to zero, the phase-field function $\phi$ converges to a step function switching values between 1 and -1 . Due to the periodicity in $y_{1}$, the interfaces appear in pairs with one increasing from -1 to 1 , which we define as the "receding" interface, followed by one decreasing from 1 to -1 , which we define as the "advancing" interface. The advancing and receding interfaces are with reference to the phase value $\phi=1$ (see Fig. 2). Thus, in the sharp-interface limit, we have $\frac{\partial\left(3 \phi-\phi^{3}\right) / 4}{\partial y_{1}} \approx \sum_{i=1}^{n}\left(\delta_{y_{r, i}(t)}-\delta_{y_{a, i}(t)}\right)$, where $\delta_{y_{r, i}(t)}$ and $\delta_{y_{a, i}(t)}$ are Dirac functions, and $y_{r, i}(t)$ and $y_{a, i}(t)$ are the $y_{1}$ coordinates of the $i$ th receding and $i$ th advancing contact points on $\partial \mathcal{G}$ at time $t$, respectively. Due to periodicity of the system, there are the same number of advancing points and receding points, and the number is $n$. Therefore, we have

$$
\begin{aligned}
& \int_{0}^{T} \int_{\partial \mathcal{G}} \cos \theta_{s}\left(y_{1}\right) \frac{\partial\left(3 \phi-\phi^{3}\right) / 4}{\partial y_{1}} d y_{1} d t \\
& \quad \approx \sum_{i=1}^{n} \int_{0}^{T}\left[\cos \theta_{s}\left(y_{r, i}(t)\right)-\cos \theta_{s}\left(y_{a, i}(t)\right)\right] d t .
\end{aligned}
$$

The equation can also be derived by asymptotic inner expansions near the moving contact points [17]. Due to the stick-slip effect of the contact point, we have $\theta_{s}\left(y_{r, i}(t)\right) \approx \theta_{b}$ and $\theta_{s}\left(y_{a, i}(t)\right) \approx \theta_{a}$ most of the time $[17,20,21]$. This leads to

$$
\begin{aligned}
& \frac{4 \sqrt{2}}{3} \mathrm{~B} U \int_{0}^{T} \int_{\partial \mathcal{G}} \cos \theta_{s}\left(y_{1}\right) \frac{\partial\left(3 \phi-\phi^{3}\right) / 4}{\partial y_{1}} d y_{1} d t \\
& \approx \frac{4 \sqrt{2}}{3} n \lambda \mathrm{B} U T .
\end{aligned}
$$

Here, $\lambda=\cos \theta_{b}-\cos \theta_{a}$ depends only on the wetting properties (Young's angles) of pore cells.

Equations (24), (25), and (27) can be combined to yield

$$
F U=U^{2} / k+F_{0} U,
$$

or equivalently a force-velocity relation

$$
U=k\left(F-F_{0}\right),
$$

where the permeability $k$ is the same as that for the homogeneous surfaces given in Eq. (21) and $F_{0}=\frac{4 \sqrt{2}}{3} n \lambda \mathrm{B} /\left(h L_{x}\right)$ is an extra depinning force due to the interface tension and chemically roughness of the surface. $\lambda=\left(\cos \theta_{b}-\cos \theta_{a}\right)$ is the wetting hysteresis property of the two-phase flow on the chemically patterned pore surface, and $\mathrm{B}$ is a dimensionless parameter of the two-phase flow (inverse of the capillary number). Therefore, the depinning force depends on the wetting and geometric properties of the pore surface as well as the capillary property of the two-phase flow. It is easy to see that the depinning force disappears if the solid surface is homogeneous so that there is no contact-angle hysteresis, i.e., $\theta_{a}=\theta_{b}$. In this case, Eq. (29) is reduced to Eq. (20).

\section{General discussions}

We note that Eqs. (20) and (29) cannot be derived from the standard Leverett model (1) for two-phase flow in porous media for the channel cases. For channel cases with periodic conditions (homogeneous or chemically patterned), the saturation $s_{i}$ does not change. Then the relative permeability $k_{r i}$ and capillary pressure $p_{c}$ in the Leverett model will be constants. 
If we suppose $\eta_{i}=1$ and ignore the gravity, then Eq. (1) is reduced to

$$
v_{i}=-k_{r i} k_{0} \nabla p_{i}, \quad i=1,2 .
$$

Notice that $\nabla p_{1}=\nabla\left(p_{2}+p_{c}\right)=\nabla p_{2}$ and is equal to $\nabla p$. Thus the total velocity (or mixture velocity as we study here)

$$
v=v_{1}+v_{2}=-\left(k_{r 1}+k_{r_{2}}\right) k_{0} \nabla p .
$$

It is easy to see that Eq. (30) derived from standard Leverett model is similar to our Eq. (20) for the homogeneous-surface case. However, there are differences for the formula of permeability. In Eq. (30) the relative permeability $k_{r i}$ depends only on the saturations. In our formula $k^{-1}=k_{0}^{-1}(1+b n)$, which depends explicitly on the number of the moving contact points, instead of the saturations.

For the chemically patterned surface, the extra depinning force is missing in Eq. (30). We note that the depinning force $F_{0}$ has a different meaning with the capillary pressure $p_{c}=$ $2 \gamma \kappa=4 \gamma \cos \theta_{e} / h$. The capillary pressure is the pressure between two fluid phases and always exists whenever $\theta_{e} \neq \frac{\pi}{2}$. The depinning force exists only when the solid surface is inhomogeneous and there is contact-angle hysteresis. Physically, the depinning force is related to the extra dissipation generated by stick-slip behavior when the solid surface is chemically patterned [18,20].

Our analysis in this paper is only for the channel pores, which corresponds to parallel layer flows in two dimensions. The generalization of the present approach to other pore geometry and three dimensions is highly nontrivial. However, the energy-dissipation relation (12) obtained from our analysis might have some hint for further studies. From Eq. (28), we have the conclusion that the dissipation

$$
\mathcal{R}=F_{0} U+k^{-1} U^{2},
$$

where the first term is the dissipation by the depinning force of contact lines due to the inhomogeneity of the solid surface, and the second term is the viscous dissipation, which includes also a contribution from the contact-line slip. We expect that, in some general cases, e.g., in a representative volume where two-phase flows mix up, the dissipation has a form similar to Eq. (31) where $F_{0}$ and $k$ might be vectors and tensors depending on the effective length of the contact lines which may also depend on the moving direction. In our understanding, it should be the projection of the moving contact lines on the transverse direction of flows. In this case, relations similar to Eqs. (20) and (29) should still hold. Similar to Eq. (22), Darcy's relation (20) becomes

$$
\nabla p \propto k^{-1} U \propto k_{0}^{-1}(1+b n) U,
$$

where $b$ depends only on the pore geometry and properties of two-phase flow, and $n$ is the effective length of contact lines in three dimensions.

The above scaling behavior (32) is consistent with the experimental results in Tallakstad et al. $[15,16]$. They observed the linear growth of the pressure jump with respect to time for a fixed velocity $U$ [or capillary number $C_{a}=\mu_{w} a^{2} v_{w} /\left(\gamma \kappa_{0}\right) \propto$ $U]$ as the front moves in the box. This means that the inverse of the permeability increases linearly. This is consistent with Eq. (32). As the front moves to the right, the total area of interfaces and the total length $n$ of the contact line increases.

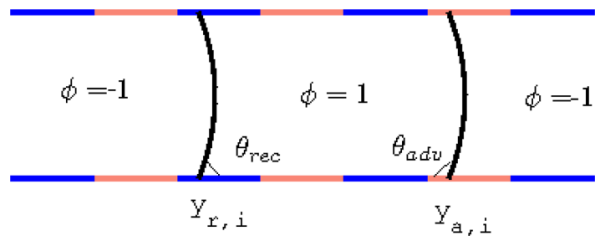

FIG. 3. (Color online) Two-phase flow in a channel with chemically patterned upper and lower boundaries.

Furthermore, they found the pressure jump $\Delta p \propto \sqrt{U}$ (or equivalently $\sqrt{C_{a}}$ ) in the steady state. This clearly implies that $k^{-1} \propto U^{-1 / 2}$ (or $k \propto \sqrt{C_{a}}$ as stated in Ref. [15]). This highly nontrivial behavior could be interpreted as the total length of contact lines $n=N l_{x}^{*} \propto C_{a}^{-1 / 2}$, where $N$ is the number of nonwetting pores and $l_{x}^{*}$ being the characteristic length of the pore in the transverse direction of the flows. The scaling is true from the statistics data on total number $N$ and the pore size $l_{x}^{*}$ in that paper. We note that the experimental results have been explained in a different way in Ref. [15].

Finally, we would say that there is still a long way to give a complete model for two-phase flow in porous media. In our analysis, we assume the two-phase flow is mixed in a representing volume and the velocity is small. The analysis will be different if there are macroscopic interfaces. For example, for a pure imbibition or a pure drainage process, the two-phase fluid is well separated and the capillary pressure will play an important role. Nevertheless, we think that the analysis for channel flow including moving contact lines might be useful in a pore-network model, which is a good start to understand more general flows in porous media.

\section{NUMERICAL VERIFICATIONS}

In this section, we carry out some numerical experiments. We solve the problem (14) and (15) by a finite difference scheme developed in Refs. [23,28]. In our numerical experiments, we set $L_{x}=100, h=40$, and $\delta=1$. The physical parameters are chosen as $R_{e}=0.03, B=12, L_{d}=5, V_{s}=5$ [23]. We set the initial velocity as $\mathbf{v}(\mathbf{y}, 0)=\mathbf{0}, p(\mathbf{y}, 0)=0$ and the initial phase function as

$$
\phi(\mathbf{y}, 0)= \begin{cases}\tanh \left(\left(0.3 L_{x}-y_{1}\right) / \sqrt{2}\right) & \text { if } y_{1} \leqslant 0.5 L_{x} \\ \tanh \left(\left(y_{1}-0.7 L_{x}\right) / \sqrt{2}\right) & \text { otherwise. }\end{cases}
$$

The periodic boundary conditions are used at the left and right boundaries. In our experiments, we use uniform meshes with
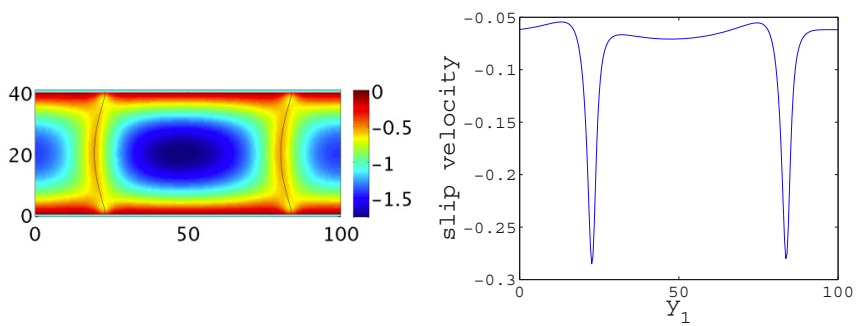

FIG. 4. (Color online) (left) A typical velocity contour and (right) slip-velocity profile. 


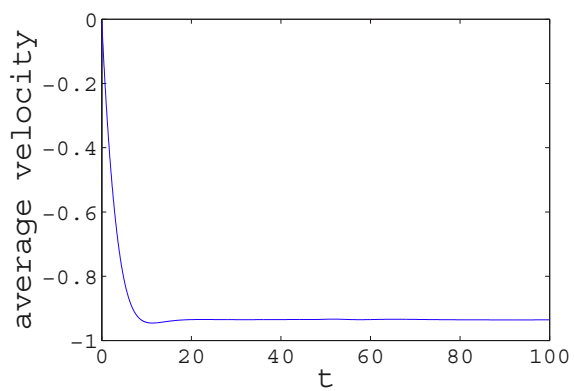

FIG. 5. (Color online) Spacial-average velocity vs time ( $l s=$ $0.4, F=-0.01)$.

space step sizes $h_{1}=100 / 256$ in the $y_{1}$ direction and $h_{2}=$ $40 / 64$ in the $y_{2}$ direction. The time step is chosen properly as $d t=(0.035 \min )\left(h_{1}, h_{2}\right)^{2} /\left(4 \mathrm{~L}_{\mathrm{d}}\right)$.

\section{A. Chemically homogeneous surface}

We consider the chemically homogeneous channel boundary with a static Young's angle $\theta_{s}=90^{\circ}$. We carry out numerical experiments for interface motion under constant force $\mathbf{F}=(F, 0)$. Different values of

$$
F=-0.03,-0.02,-0.01,-0.005,-0.0025,-0.00125
$$

and different slip length $l_{s}=3.2,1.6,0.8,0.4$ are used.

Figure 4 shows a typical horizontal velocity contour and the slip velocity profile when slip length $l_{s}=0.4$ and the force $F=0.01$. It is clearly seen that the slip region near the contact line will contribute significantly to the integral in the right hand side of Eq. (18). Figure 5 shows the averaged horizontal velocity $U$ as a function of time. It is easy to see that the horizontal velocity becomes constant after some time. The averaged vertical velocity is always zero and is not shown here. This implies that the flow tends to a traveling wave.

The force-velocity relation $U=k F$ is clearly verified in Fig. 6. The estimated permeability $k$ in each case is shown in the first row of Table I. In the second row, we show the permeability for the one-phase channel computed by $k_{0}=$ $h\left(h+6 l_{s}\right) / 12$. It is easy to see that the permeability for the two-phase flow is significantly reduced compared with that of the one-phase flow, in agreement with our analysis.

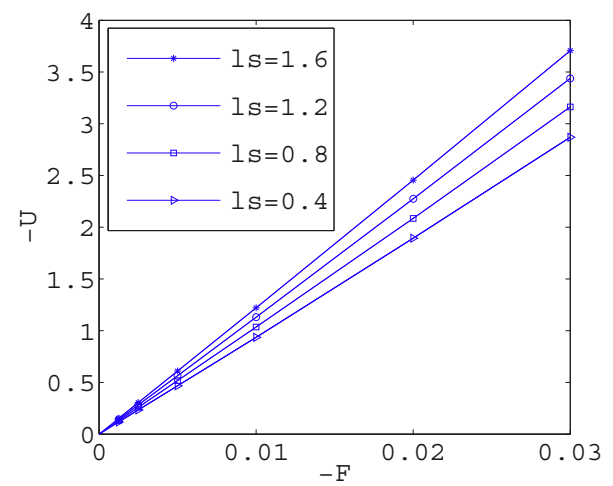

FIG. 6. (Color online) Darcy's relation for two-phase channel flow with homogeneous boundary.
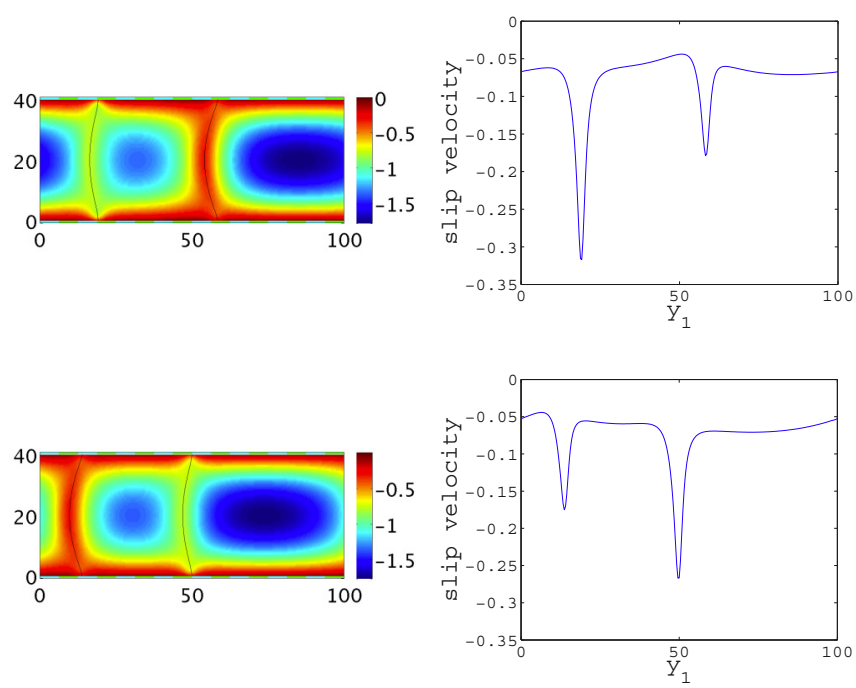

FIG. 7. (Color online) Some typical velocity contours and slipvelocity profile $\left(\theta_{s}=80^{\circ}, F=-0.01\right)$.

\section{B. Chemically patterned surface}

We also carry out numerical experiments for a chemically patterned channel boundary. We assume that the top and bottom boundaries have the same periodic pattern as shown in Fig. 3 with the static Young's angles $\theta_{s}$ and $\pi-\theta_{s}$, respectively. We consider two cases for $\theta=80^{\circ}$ and $\theta=60^{\circ}$. Similar to the homogeneous case, we simulate interface motion under different external force. In these computations, we set $l_{s}=0.4$.

Figure 7 shows some typical horizontal velocity contour and the slip-velocity profile when $\theta_{s}=80^{\circ}$ and the force $F=$ 0.01 . The large slip behavior near the contact line is clearly shown again. Figure 8 shows the advancing contact angle and receding contact angle as a function of time. It is clearly seen that the advancing contact angle is oscillating around $180^{\circ}-$ $\theta_{s}=100^{\circ}$, and the receding angle is changing around $\theta_{s}=$ $80^{\circ}$. Figure 9 shows the space-averaged horizontal velocity $\tilde{U}(t)=\int_{\mathcal{B}} v_{1} d y_{1} d y_{2}$ as a function of time. It is easy to see that $\tilde{U}(t)$ becomes periodic in time. The averaged vertical velocity is always zero (not shown here). This implies that the flow becomes periodic in time. In Fig. 10, we show the relation between the values of the averaged velocity $U$ (averaged in

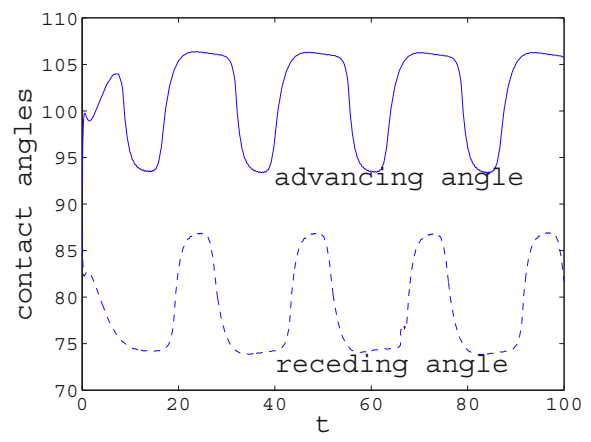

FIG. 8. (Color online) Advancing contact angle and receding contact angle $\left(\theta_{s}=80^{\circ}, F=-0.01\right)$. 


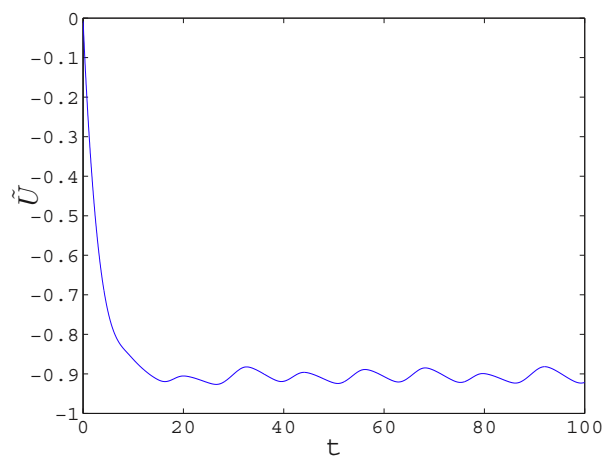

FIG. 9. (Color online) Spacial-average velocity vs time $\left(\theta_{s}=\right.$ $\left.80^{\circ}, F=-0.01\right)$.

both space and time) and the values of the external force $F$ for two different contact angles. The non-Darcy behavior (29) is clearly shown. In this experiment, when $F$ is too small, the numerical errors will affect the numerical results and make values slightly deviate from the theoretical predictions.

\section{CONCLUSION}

In conclusion, channel flows constitute probably the most elementary component of any considerations about flows in porous media. Hence the permeability dependence on moving contact lines and the non-Darcy behavior induced by chemical or geometric roughness should persist in the general problem of multiphase permeability of porous media. In particular, our result offers a quantitatively understanding of the prior observation of non-Darcy behavior in either simulations or experiments [18-22] that involved contact line motion. Here the extra force $F_{0}$ can be especially important at low flow rates, representing the dissipation caused by the contact lines when each crosses from one region of the contact angle to another. Such considerations have been missing in previous

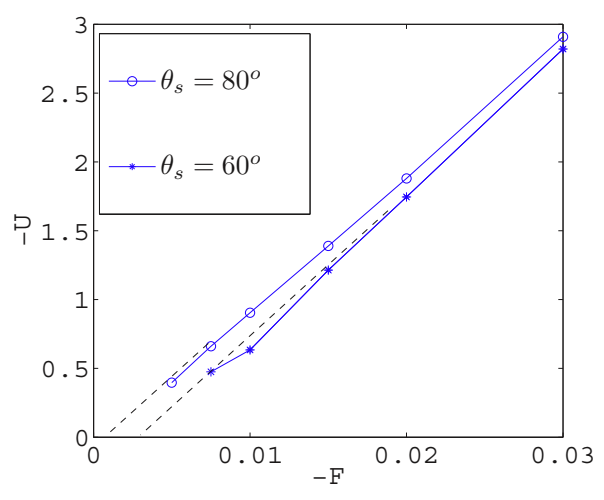

FIG. 10. (Color online) Force-velocity relation for two-phase channel flow with periodic patterned boundary.

considerations of multiphase permeability, and its inclusion may enhance the realism of the modeling effort. Finally, the generalization of the analysis in this paper to other pore geometry and three-dimensional models is highly nontrivial. We expect that the energy dissipation relation and the analysis for channel flows might be helpful in a simple pore-network model.

\section{ACKNOWLEDGMENTS}

We thank Ping Sheng for many illuminating discussions. This publication was based on work supported in part by Hong Kong RGC-GRF Grants No. 605513, No. 605311, and by NSFC 11001260.

\section{APPENDIX A: CALCULATIONS}

\section{The expansions}

Substituting the expansions in Sec. II B into Eqs. (7) and (8), we have

$$
\begin{aligned}
& \frac{1}{\varepsilon} \frac{\partial}{\partial s}\left(\phi_{0}+\varepsilon \phi_{1}+\cdots\right)+\left(\mathbf{v}_{0}+\varepsilon \mathbf{v}_{1}+\cdots\right) \cdot\left(\nabla_{x}+\varepsilon^{-1} \nabla_{y}\right)\left(\phi_{0}+\varepsilon \phi_{1}+\cdots\right) \\
& \quad=\varepsilon^{2} L_{d}\left[\Delta_{x}+2 \varepsilon^{-1}\left(\nabla_{x} \cdot \nabla_{y}\right)+\varepsilon^{-2} \Delta_{y}\right]\left(\varepsilon^{-1} \mu_{0}+\mu_{1}+\cdots\right), \\
& \varepsilon \mathbf{R}_{e}\left[\frac{1}{\varepsilon} \frac{\partial \mathbf{v}}{\partial s}+\left(\mathbf{v}_{0}+\varepsilon \mathbf{v}_{1}+\cdots\right)\left(\nabla_{x}+\frac{1}{\varepsilon} \nabla_{y}\right)\left(\mathbf{v}_{0}+\varepsilon \mathbf{v}_{1}+\cdots\right)\right] \\
& =\mathbf{F}-\left(\nabla_{x}+\varepsilon^{-1} \nabla_{y}\right)\left(p_{0}+\varepsilon p_{1}+\cdots\right)+\varepsilon^{2}\left[\Delta_{x}+2 \varepsilon^{-1}\left(\nabla_{x} \cdot \nabla_{y}\right)+\varepsilon^{-2} \Delta_{y}\right]\left(\mathbf{v}_{0}+\varepsilon \mathbf{v}_{1}+\cdots\right) \\
& \quad+\varepsilon^{2} \mathrm{~B}\left(\varepsilon^{-1} \mu_{0}+\mu_{1}+\cdots\right)\left(\nabla_{x}+\varepsilon^{-1} \nabla_{y}\right)\left(\phi_{0}+\varepsilon \phi_{1}+\cdots\right), \\
& \left(\nabla_{x}+\varepsilon^{-1} \nabla_{y}\right) \cdot\left(\mathbf{v}_{0}+\varepsilon \mathbf{v}_{1}+\cdots\right)=0,
\end{aligned}
$$

with boundary conditions

$$
\begin{aligned}
-\mathrm{V}_{\mathbf{s}}\left(\mathcal{L}_{0}\left(\phi_{0}\right)+O(\varepsilon)\right) & =\varepsilon\left[\frac{1}{\varepsilon} \frac{\partial}{\partial s}\left(\phi_{0}+\varepsilon \phi_{1}+\cdots\right)\left(v_{0, \tau}+\varepsilon v_{1, \tau}+\cdots\right)\left(\nabla_{x}+\varepsilon^{-1} \nabla_{y}\right)\left(\phi_{0}+\varepsilon \phi_{1}+\cdots\right) \cdot \tau\right], \\
l_{s}^{-1}\left(v_{0, \tau}+\varepsilon v_{1, \tau}+\cdots\right) & =-\varepsilon\left(\nabla_{x}+\varepsilon^{-1} \nabla_{y}\right)\left(v_{0, \tau}+\varepsilon v_{1, \tau}+\cdots\right) \cdot \mathbf{n}+\varepsilon \mathrm{B}\left(\mathcal{L}_{0}\left(\phi_{0}\right)+O(\varepsilon)\right)\left(\nabla_{x}+\varepsilon^{-1} \nabla_{y}\right)\left(\phi_{0}+\varepsilon \phi_{1}+\cdots\right) \cdot \tau, \\
0 & =\left(\nabla_{x}+\varepsilon^{-1} \nabla_{y}\right)\left(\varepsilon^{-1} \mu_{0}+\mu_{1}+\cdots\right) \cdot \mathbf{n}, \\
0 & =\left(\mathbf{v}_{0}+\varepsilon \mathbf{v}_{1}+\cdots\right) \cdot \mathbf{n} .
\end{aligned}
$$

The leading orders of the equation gives Eqs. (9)-(11). 


\section{Derivation of dissipation function}

We now multiply by $\mathbf{v}_{0}$ the first equation of Eq. (10) and integrate over $\mathcal{B}$. This leads to

$$
\begin{aligned}
\int_{\mathcal{B}} \mathrm{R}_{\mathrm{e}} & {\left[\frac{\partial \mathbf{v}_{0}}{\partial s} \cdot \mathbf{v}_{0}+\left(\mathbf{v}_{0} \cdot \nabla_{y}\right) \mathbf{v}_{0} \cdot \mathbf{v}_{0}\right] d \mathbf{y} } \\
= & {[\mathbf{F}-\nabla P(\mathbf{x})] \cdot \int_{\mathcal{B}} \mathbf{v}_{0} d \mathbf{y}+\int_{\mathcal{B}}\left[-\nabla_{y} p_{1} \cdot \mathbf{v}_{0}+\Delta_{y} \mathbf{v}_{0} \cdot \mathbf{v}_{0}\right.} \\
& \left.+\mathrm{B} \mu_{0} \nabla_{y} \phi_{0} \cdot \mathbf{v}_{0}\right] d \mathbf{y} .
\end{aligned}
$$

We have used $\nabla P(x)=\nabla_{x} p_{0}(x)$. The first term on the lefthand side is given by

$$
\mathrm{R}_{\mathrm{e}} \int_{\mathcal{B}} \frac{\partial \mathbf{v}_{0}}{\partial s} \cdot \mathbf{v}_{0} d \mathbf{y}=\frac{\mathrm{R}_{\mathrm{e}}}{2} \frac{\partial}{\partial s} \int_{\mathcal{B}}\left|\mathbf{v}_{0}\right|^{2} d \mathbf{y} .
$$

The second term on the left-hand side of Eq. (A3) is calculated as

$$
\begin{aligned}
\mathrm{R}_{\mathrm{e}} & \int_{\mathcal{B}}\left(\mathbf{v}_{0} \cdot \nabla_{y}\right) \mathbf{v}_{0} \cdot \mathbf{v}_{0} d \mathbf{y} \\
= & \frac{\mathrm{R}_{\mathrm{e}}}{2} \int_{\mathcal{B}}\left(\mathbf{v}_{0} \cdot \nabla_{y}\right)\left|\mathbf{v}_{0}\right|^{2} d \mathbf{y} \\
= & \frac{\mathrm{R}_{\mathrm{e}}}{2} \int_{\partial \mathcal{B}}\left(\mathbf{v}_{0} \cdot \mathbf{n}\right)\left|\mathbf{v}_{0}\right|^{2} d \sigma_{y}-\frac{\mathrm{R}_{\mathrm{e}}}{2} \int_{\partial \mathcal{B}}\left(\nabla_{y} \cdot \mathbf{v}_{0}\right)\left|\mathbf{v}_{0}\right|^{2} d \mathbf{y}=0 .
\end{aligned}
$$

Here, $d \sigma_{y}$ is the line integral variable along the boundary of $\partial \mathcal{G}$. In the derivation, we use the divergence-free condition of $\mathbf{v}_{0}$, the zero normal velocity boundary condition on $\partial \mathcal{G}$, and the periodic boundary condition of $\mathbf{v}_{0}$ on $\partial \mathcal{Y}$. Similarly, the second term on the right-hand side of Eq. (A3) is given by

$$
\begin{aligned}
& -\int_{\mathcal{B}} \nabla_{y} p_{1} \cdot \mathbf{v}_{0} d \mathbf{y} \\
& \quad=-\int_{\partial \mathcal{B}} p_{1} \mathbf{v}_{0} \cdot \mathbf{n} d \sigma_{y}+\int_{\mathcal{B}} p_{1} \nabla_{y} \cdot \mathbf{v}_{0} d \mathbf{y}=0 .
\end{aligned}
$$

The third term on the right-hand side of Eq. (A3) is given by

$$
\begin{aligned}
\int_{\mathcal{B}} \Delta_{y} \mathbf{v}_{0} \cdot \mathbf{v}_{0} d \mathbf{y}= & \int_{\partial \mathcal{B}}\left(\mathbf{n} \cdot \nabla_{y}\right) \mathbf{v}_{0} \cdot \mathbf{v}_{0} d \sigma_{y}-\int_{\mathcal{B}}\left|\nabla_{y} \mathbf{v}_{0}\right|^{2} d \mathbf{y} \\
= & \int_{\partial \mathcal{G}}\left(\nabla_{y} v_{0, \tau} \cdot \mathbf{n}\right) v_{0, \tau} d \sigma_{y}-\int_{\mathcal{B}}\left|\nabla_{y} \mathbf{v}_{0}\right|^{2} d \mathbf{y} \\
= & -l_{s}^{-1} \int_{\partial \mathcal{G}} v_{0, \tau}^{2} d \sigma_{y}+\mathrm{B} \int_{\partial \mathcal{G}} \mathcal{L}_{0}\left(\phi_{0}\right) \nabla_{y} \phi_{0} \\
& \cdot \tau v_{0, \tau} d \sigma_{y}-\int_{\mathcal{B}}\left|\nabla_{y} \mathbf{v}_{0}\right|^{2} d \mathbf{y}
\end{aligned}
$$

Here, we use the slip boundary conditions in Eq. (11) in addition to the other boundary conditions of $\mathbf{v}_{0}$. The second term in Eq. (A7) could be further computed as

$$
\begin{aligned}
& \mathrm{B} \int_{\partial \mathcal{G}} \mathcal{L}_{0}\left(\phi_{0}\right) \nabla_{y} \phi_{0} \cdot \tau v_{0, \tau} d \sigma_{y} \\
& =\mathrm{B} \int_{\partial \mathcal{G}} \mathcal{L}_{0}\left(\phi_{0}\right)\left(\dot{\phi}_{0}-\frac{\partial \phi_{0}}{\partial s}\right) d \sigma_{y} \\
& =-\frac{\mathrm{B}}{\mathrm{V}_{s}} \int_{\partial \mathcal{G}} \dot{\phi}_{0}^{2} d \sigma_{y}-\mathrm{B} \int_{\partial \mathcal{G}} \mathcal{L}_{0}\left(\phi_{0}\right) \frac{\partial \phi_{0}}{\partial s} d \sigma_{y} .
\end{aligned}
$$

The last term on the right-hand side of Eq. (A3) is calculated as

$$
\begin{aligned}
\int_{\mathcal{B}} \mathrm{B} & \mu_{0} \nabla_{y} \phi_{0} \cdot \mathbf{v}_{0} d \mathbf{y} \\
= & \int_{\mathcal{B}} \mathrm{B} \mu_{0}\left(\mathrm{~L}_{\mathrm{d}} \Delta_{y} \mu_{0}-\frac{\partial \phi_{0}}{\partial s}\right) d \mathbf{y} \\
= & \mathrm{BL} \int_{d \mathcal{B}} \mu_{0} \nabla_{y} \mu_{0} \cdot \mathbf{n} d \sigma_{y}-\mathrm{B} \mathrm{L}_{\mathrm{d}} \int_{\partial \mathcal{B}}\left|\nabla \mu_{0}\right|^{2} d \mathbf{y} \\
& -\mathrm{B} \int_{\mathcal{B}} \mu_{0} \frac{\partial \phi_{0}}{\partial s} d \mathbf{y} \\
= & -\mathrm{BL} \int_{d}\left|\nabla \mu_{0}\right|^{2} d \mathbf{y}-\mathrm{B} \int_{\mathcal{B}} \mu_{0} \frac{\partial \phi_{0}}{\partial s} d \mathbf{y} .
\end{aligned}
$$

Finally, direct computations gives

$$
\begin{aligned}
& -\mathrm{B} \int_{\partial \mathcal{G}} \mathcal{L}_{0}\left(\phi_{0}\right) \frac{\partial \phi_{0}}{\partial s} d \sigma_{y} \\
& =-\mathrm{B} \delta \int_{\partial \mathcal{G}} \nabla_{y} \phi_{0} \cdot \mathrm{n} \frac{\partial \phi_{0}}{\partial s} d \sigma_{y}-\mathrm{B} \frac{\partial}{\partial s} \int_{\partial \mathcal{G}} \gamma_{w f}\left(\phi_{0}\right) d \sigma_{y} \\
& -\mathrm{B} \int_{\mathcal{B}} \mu_{0} \frac{\partial \phi_{0}}{\partial s} d \mathbf{y} \\
& =\mathrm{B} \delta \int_{\partial \mathcal{G}} \nabla_{y} \phi_{0} \cdot \mathrm{n} \frac{\partial \phi_{0}}{\partial s} d \sigma_{y}-\frac{\mathrm{B} \delta}{2} \frac{\partial}{\partial s} \int_{\mathcal{B}}\left|\nabla_{y} \phi_{0}\right|^{2} d \mathbf{y} \\
& -\frac{\mathrm{B}}{\delta} \frac{\partial}{\partial s} \int_{\mathcal{B}} \frac{\left(1-\phi_{0}^{2}\right)^{2}}{4} d \mathbf{y} .
\end{aligned}
$$

Combining all the terms, we have

$$
\begin{aligned}
{[\mathbf{F}-} & \nabla P(\mathbf{x})] \cdot \int_{\mathcal{B}} \mathbf{v}_{0} d \mathbf{y} \\
= & \int_{\mathcal{B}}\left|\nabla \mathbf{v}_{0}\right|^{2} d \mathbf{y}+\int_{\partial \mathcal{G}} \frac{v_{0, \tau}^{2}}{l_{s}} d \sigma_{y}+\frac{\mathrm{B}}{\mathrm{V}_{\mathrm{s}}} \int_{\partial \mathcal{G}} \dot{\phi}_{0}^{2} d \sigma_{y} \\
& +\mathrm{B} \mathrm{L}_{\mathrm{d}} \int_{\mathcal{B}}\left|\nabla \mu_{0}\right| d \mathbf{y}+\frac{\partial}{\partial s}\left[\mathrm{R}_{\mathrm{e}} \int_{\mathcal{B}} \frac{\left|\mathbf{v}_{0}\right|^{2}}{2} d \mathbf{y}\right. \\
& \left.+\mathrm{B} \int_{\partial \mathcal{G}} \gamma_{w f}\left(\phi_{0}\right) d \sigma_{y}+\mathrm{B} \int_{\mathcal{B}}\left(\frac{\delta}{2}\left|\nabla \phi_{0}\right|^{2}+\frac{1}{\delta} f\left(\phi_{0}\right)\right) d \mathbf{y}\right],
\end{aligned}
$$

with $f(\phi)=\left(1-\phi^{2}\right)^{2} / 4$. We then integrate the above equation in fast time $s$ in $(0, T)$ and, noticing the assumption of time periodicity, we have Eq. (12).

\section{Computations in homogeneous channel}

We multiply by $\mathbf{U}$ the first equation of Eqs. (16) and integrate over $\mathcal{B}$. We have

$$
\begin{aligned}
\mathbf{F} \cdot \mathbf{U}|\mathcal{B}|= & \mathrm{R}_{\mathrm{e}} \int_{\mathcal{B}}[(\mathbf{v}-\mathbf{U}) \cdot \nabla] \mathbf{v} \cdot \mathbf{U} d \mathbf{y} \\
& -\int_{\mathcal{B}}(-\nabla p+\Delta \mathbf{v}+\mathrm{B} \mu \nabla \phi) \cdot \mathbf{U} d \mathbf{y} .
\end{aligned}
$$


The first term on the right-hand side becomes

$$
\begin{aligned}
& \mathrm{R}_{\mathrm{e}} \int_{\mathcal{B}}[(\mathbf{v}-\mathbf{U}) \cdot \nabla] \mathbf{v} \cdot \mathbf{U} d \mathbf{y} \\
& =\mathrm{R}_{\mathrm{e}} U \int_{\mathcal{B}}(\mathbf{v}-\mathbf{U}) \cdot \nabla v_{1} d \mathbf{y} \\
& =-\mathrm{R}_{\mathrm{e}} U \int_{\mathcal{B}} v_{1} \boldsymbol{\nabla} \cdot \mathbf{v} d \mathbf{y}+\mathrm{R}_{\mathrm{e}} U \int_{\partial \mathcal{G}}(\mathbf{v}-\mathbf{U}) \cdot \mathbf{n} v_{1} d \sigma_{y} \\
& \quad+\mathrm{R}_{e} U\left(\int_{\left\{y_{1}=L_{x}\right\}}\left(v_{1}-U\right) v_{1} d y_{2}\right. \\
& \left.\quad-\int_{\left\{y_{1}=0\right\}}\left(v_{1}-U\right) v_{1} d y_{2}\right)=0 .
\end{aligned}
$$

Here, we have used the periodic condition of $\mathbf{v}$ at $y_{1}=0, L_{x}$ in addition to the boundary condition of $\mathbf{v}$ on $\partial \mathcal{G}$ as well as the divergence-free property of $\mathbf{v}$ in $\mathcal{B}$. Integrating by parts again, the second term of the right-hand side of Eq. (A13) reads

$$
\begin{aligned}
\int_{\mathcal{B}} \nabla p \cdot \mathbf{U} d \mathbf{y}= & \int_{\partial \mathcal{G}} p \mathbf{U} \cdot \mathbf{n} d \sigma_{y}+\int_{\left\{y_{1}=L_{x}\right\}} p U d y_{2} \\
& -\int_{\left\{y_{1}=0\right\}} p U d y_{2}=0 .
\end{aligned}
$$

This is simply from the periodic boundary condition of $p$ at $y_{1}=0, L_{x}$. The third term of the right-hand side of Eq. (A13) gives

$$
\begin{aligned}
-\int_{\mathcal{B}} \Delta \mathbf{v} \cdot \mathbf{U} d \mathbf{y}= & -\int_{\partial \mathcal{G}} \frac{\partial \mathbf{v}}{\partial \mathbf{n}} \cdot \mathbf{U} d s_{y}-\int_{\left\{y_{1}=L_{x}\right\}} \frac{\partial \mathbf{v}}{\partial \mathbf{n}} \cdot \mathbf{U} d y_{2} \\
& -\int_{\left\{y_{1}=0\right\}} \frac{\partial \mathbf{v}}{\partial \mathbf{n}} \cdot \mathbf{U} d y_{2} \\
= & -U \int_{\partial \mathcal{G}} \frac{\partial v_{\tau}}{\partial \mathbf{n}} d \sigma_{y} \\
= & U \int_{\partial \mathcal{G}} \frac{v_{\tau}}{l_{s}} d \sigma_{y}-\mathrm{B} U \int_{\partial \mathcal{G}} L(\phi) \frac{\partial \phi}{\partial \tau} d \sigma_{y} \\
= & U \int_{\partial \mathcal{G}} \frac{v_{\tau}}{l_{s}} d \sigma_{y}-\delta \mathrm{B} U \int_{\partial \mathcal{G}} \frac{\partial \phi}{\partial \mathbf{n}} \frac{\partial \phi}{\partial \tau} d \sigma_{y} \\
& -\frac{\sqrt{2} \mathrm{~B} U \cos \theta_{s}}{6} \int_{\partial \mathcal{G}} \frac{\partial\left(3 \phi-\phi^{3}\right)}{\partial y_{1}} d y_{1} \\
= & U \int_{\partial \mathcal{G}} \frac{v_{\tau}}{l_{s}} d \sigma_{y}-\delta \mathrm{B} U \int_{\partial \mathcal{G}} \frac{\partial \phi}{\partial \mathbf{n}} \frac{\partial \phi}{\partial \tau} d \sigma_{y} .
\end{aligned}
$$

Here in the second equation, we use the periodic condition of $\mathbf{v}$ on $y_{1}=0, L_{x}$. In the third equation, we have used the generalized Navier condition on $\partial \mathcal{G}$, and in the fourth equation we have used the fact that $\theta_{s}$ is a constant on the homogeneous surface and $\phi$ is periodic. The last term in equation (A13) can be calculated as

$$
\begin{aligned}
& -\mathrm{B} \int_{\mathcal{B}} \mu \nabla \phi \cdot \mathbf{U} d \mathbf{y} \\
& =-\mathrm{B} U \int_{\mathcal{B}}\left(-\delta \Delta \phi-\frac{\phi-\phi^{3}}{\delta}\right) \frac{\partial \phi}{\partial y_{1}} d \mathbf{y}
\end{aligned}
$$

$$
\begin{aligned}
= & \delta \mathrm{B} U \int_{\partial \mathcal{G}} \frac{\partial \phi}{\partial \mathbf{n}} \frac{\partial \phi}{\partial y_{1}} d \sigma_{y}-\frac{\mathrm{B} U}{\delta} \int_{\mathcal{B}} \delta^{2} \nabla \phi \cdot \nabla \partial_{y_{1}} \phi \\
& +\left(\phi-\phi^{3}\right) \partial_{y_{1}} \phi d \mathbf{y} \\
= & \delta \mathrm{B} U \int_{\partial \mathcal{G}} \frac{\partial \phi}{\partial \mathbf{n}} \frac{\partial \phi}{\partial \tau} d \sigma_{y}-\frac{\mathrm{B} U}{\delta} \int_{\mathcal{B}} \delta^{2} \partial_{y_{1}}|\nabla \phi|^{2} \\
& +\partial_{y_{1}} \frac{\left(1-\phi^{2}\right)^{2}}{4} d \mathbf{y} \\
= & \delta \mathrm{B} U \int_{\partial \mathcal{G}} \frac{\partial \phi}{\partial \mathbf{n}} \frac{\partial \phi}{\partial \tau} d \sigma_{y} .
\end{aligned}
$$

Combining Eqs. (A13)-(A17), we have

$$
\mathbf{F} \cdot \mathbf{U}|\mathcal{B}|=U \int_{\partial \mathcal{G}} l_{s}^{-1} v_{\tau} d \sigma_{y} .
$$

This further leads to Eq. (18).

\section{Computations in chemically patterned channel}

We first estimate each term on the right-hand side of Eq. (23). The computations are similar to that in the previous section. Direct computations give

$$
\begin{aligned}
& \operatorname{Re} \int_{\mathcal{B}}\left[\partial_{t} \mathbf{v} \cdot \mathbf{U}+(\mathbf{v} \cdot \nabla) \mathbf{v} \cdot \mathbf{U}\right] d \mathbf{y} \\
& =\operatorname{Re} U \int_{\mathcal{B}} \partial_{t} v_{1} d \mathbf{y} \\
& =\operatorname{Re} U \partial_{t} \int_{\mathcal{B}} v_{1} d \mathbf{y} .
\end{aligned}
$$

For the second term, we have

$$
\int_{\mathcal{B}} \nabla p \cdot \mathbf{U} d \mathbf{y}=0 .
$$

For the third term, we have

$$
\begin{aligned}
-\int_{\mathcal{B}} \Delta \mathbf{v} \cdot \mathbf{U} d \mathbf{y}= & -U \int_{\partial \mathcal{G}} \frac{\partial v_{\tau}}{\partial \mathbf{n}} d \sigma_{y} \\
= & U \int_{\partial \mathcal{G}} l_{s}^{-1} v_{\tau} d \sigma_{y}-\delta \mathrm{B} U \int_{\partial \mathcal{G}} \frac{\partial \phi}{\partial \mathbf{n}} \frac{\partial \phi}{\partial \tau} d \sigma_{y} \\
& -\frac{\sqrt{2}}{6} \mathrm{~B} U \int_{\partial \mathcal{G}} \cos \theta_{s}\left(y_{1}\right) \frac{\partial\left(3 \phi-\phi^{3}\right)}{\partial y_{1}} d y_{1} .
\end{aligned}
$$

The last term in Eq. (23) yields

$$
-\mathrm{B} \int_{\mathcal{B}} \mu \nabla \phi \cdot \mathbf{U} d \mathbf{y}=\delta \mathrm{B} U \int_{\partial \mathcal{G}} \frac{\partial \phi}{\partial \mathbf{n}} \frac{\partial \phi}{\partial \tau} d \sigma_{y} .
$$

Combining Eqs. (A19)-(A22), we have

$$
\begin{aligned}
\mathbf{F} \cdot \mathbf{U}|\mathcal{B}|= & \mathrm{R}_{e} U \partial_{t} \int_{\mathcal{B}} v_{1} d \mathbf{y}+U \int_{\partial \mathcal{G}} l_{s}^{-1} v_{\tau} d \sigma_{y} \\
& -\frac{\sqrt{2}}{6} \mathrm{~B} U \int_{\partial \mathcal{G}} \cos \theta_{s}\left(y_{1}\right) \frac{\partial\left(3 \phi-\phi^{3}\right)}{\partial y_{1}} d y_{1} .
\end{aligned}
$$

Integrating the above equation over time over one period, the left-hand side gives

$$
\int_{0}^{T} \mathbf{F} \cdot \mathbf{U}|\mathcal{B}| d t=F U|\mathcal{B}| T=F U L_{x} h T .
$$




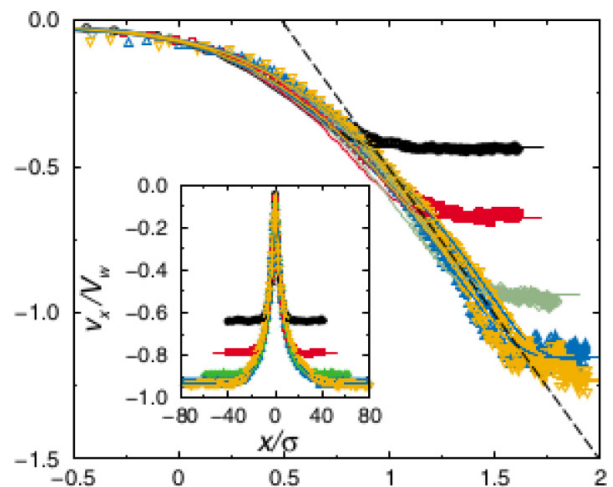

FIG. 11. (Color online) Scaled slip velocity vs scaled distance from MCL: the log-log scaling and the original profile (inset) [25].

The first term on the right-hand side of the Eq. (A23) gives

$$
\mathrm{R}_{e} U \int_{0}^{T}\left(\partial_{t} \int_{\mathcal{B}} v_{1} d \mathbf{y}\right) d t=0,
$$

because of the time-periodicity of the velocity. This leads to Eq. (24).

\section{APPENDIX B: SLIP PROFILE NEAR MOVING CONTACT LINE}

Far from the moving contact line, it is well known that the slip velocity of fluid is relatively small so that a no-slip boundary condition is a good approximation in general. In the vicinity of the moving contact line, near-complete slip has been observed by molecular dynamics (MD) simulations for immiscible flows (see Ref. [25] and references therein). The slip profile is studied in Ref. [25] by MD simulations and by continuum simulations based on the phase-field model (7) with GNBC boundary conditions. It is found that there is a partial-slip region spreading from the complete-slip region to the nearly-no-slip region. In the partial-slip region, the ratio of the slip velocity and the macroscopic velocity, $v_{\text {slip }} / U$, has a universal profile which decays in a power law of $1 / x$ [see Fig. 11] where $x$ is the distance from the moving contact line (MCL). The outer cutoff for the partial-slip region, denoted by $R$, is determined by the overall size of the system (the channel height $h$ in our case). The partial-slip region has a significant contribution to total dissipations. It is also shown that the universal slip velocity $v_{\text {slip }}$ can be approximated by

$$
\frac{v_{\text {slip }}}{U}=\frac{1}{1+x /\left(a l_{s}\right)},
$$

where $a=2.14$ is obtained by a data fitting approach to the MD experiment results. Based on this, in the integral on the right-hand side of Eq. (18), the slip region will contribute a constant:

$$
\mathrm{A}=2 l_{s}^{-1} \int_{0}^{\mathrm{R}} \frac{v_{\text {slip }}}{U} d x=2 \times 2.14 \ln \left(1+R /\left(2.14 l_{s}\right)\right),
$$

which could be very large. For example, if $l_{s}=1 \mathrm{~nm}$ and $\mathrm{R}=1 \mu \mathrm{m}$, then $\mathrm{A} \approx 36.2$. This might change the permeability significantly, especially when $L_{x} / h$ is not too big.

Hocking gave an asymptotic analysis to the moving contact line problem [26] assuming the Navier slip boundary condition

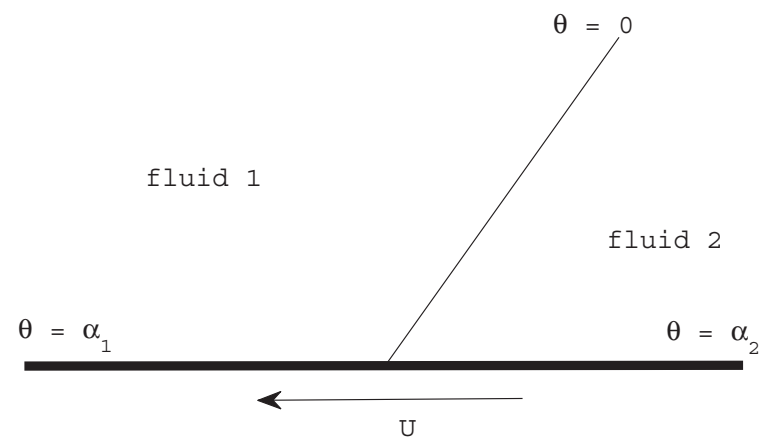

FIG. 12. Two-phase fluid with a moving contact line.

near MCL, in which the partial-slip profile with a power law $1 / x$ is also obtained. The author considered two-phase fluids on a moving solid surface as in Fig. 12. The solid surface has a constant velocity $U$ and the contact line does not move with the boundary. Away from the interface, the system is assumed to be described by the Stokes equation with the Navier-slip boundary condition. This can also be derived from the sharp-interface limit of system (14) and (15) [29]. The inner region is considered by setting $\rho=\ln \left(x / l_{s}\right)$. With the help of stream functions, the author derived the following formula for tangential stress for the two fluids [that is, Eq. (2.7) in Ref. [26]],

$$
\tau_{1}=\eta_{1} U x^{-1} k_{1}(\rho), \quad \tau_{2}=\eta_{2} U x^{-1} k_{2}(\rho),
$$

where $\eta_{1}$ and $\eta_{2}$ are viscosities for the two fluids, $k_{1}$ and $k_{2}$ are two functions determined by two coupled integral equations [see Eq. (2.19) in Ref. [26] for the formula]. $k_{1}$ and $k_{2}$ satisfy the following boundary conditions:

$$
\begin{aligned}
& k_{i}(\rho) \sim e^{\rho} \text { as } \rho \rightarrow-\infty, \quad i=1,2, \\
& k_{i}(\rho) \rightarrow \hat{k}_{i} \text { as } \rho \rightarrow+\infty, \quad i=1,2,
\end{aligned}
$$

where $\hat{k}_{i}$ are given constants determined by the contact angles and the viscosity ratio. In addition, when the slip region $R$ is large, the friction force due to the moving contact line is approximated by [Eq. (2.28) in Ref. [26]]

$$
f_{i}(\mathrm{R})=\int_{0}^{\mathrm{R}} \tau_{i} d x=\eta_{i} U\left(\hat{k}_{i} \ln \left(\mathrm{R} / l_{s}\right)+h_{i}+O(1)\right)
$$

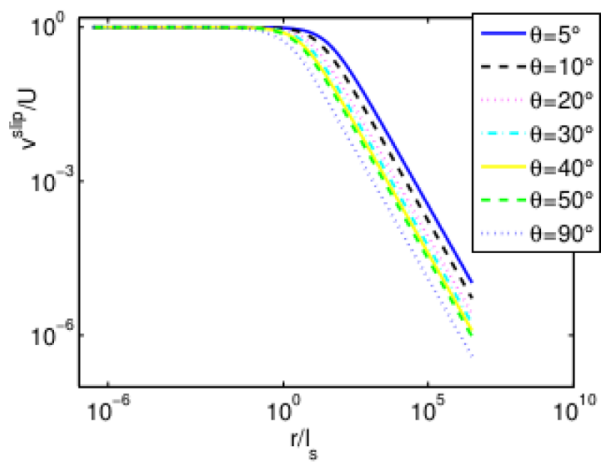

FIG. 13. (Color online) Scaled slip velocity vs scaled distance from MCL given by asymptotics [27]. 
Here, $h_{i}$ are two values that depend on contact angles, viscosity, etc. Some specific values can be found in Table I in Ref. [26].

Noticing the Navier slip boundary condition $l_{s}^{-1} v_{i \text {,slip }}=\tau_{i}$, we derive from (B3) that

$$
\frac{v_{i, \text { slip }}}{U}=\frac{l_{s}}{x} \eta_{i} k_{i}\left(\ln \frac{x}{l_{s}}\right), \quad i=1,2 .
$$

This implies the slip velocity has a profile given by function $k_{i}$. The integral equations for $k_{i}$ are solved numerically in Ref. [27] for a liquid-gas system and Fig. 13 displays the scaled slip velocity and the scaled distance $x / l_{s}$ in the inner region of MCL for the liquid phase and for different contact angles. The power-law behavior of $v_{\text {slip }} / U \propto l_{s} / x$ is clearly shown. From Eq. (B4), we can compute the parameter A as

$$
\begin{aligned}
\mathrm{A} & =\frac{1}{U}\left(\int_{0}^{\mathrm{R}} l_{s}^{-1} v_{1, \mathrm{slip}} d x+\int_{0}^{\mathrm{R}} l_{s}^{-1} v_{2, \mathrm{slip}} d x\right) \\
& =\frac{1}{U}\left(\int_{0}^{\mathrm{R}} \tau_{1} d x+\int_{0}^{\mathrm{R}} \tau_{2} d x\right) \\
& =\sum_{i=1,2} \eta\left(\hat{k}_{i} \ln \left(\mathrm{R} / l_{s}\right)+h_{i}+O(1)\right) .
\end{aligned}
$$

When $\mathrm{R}$ is relatively large compared to $l_{s}$, A can be significantly large, just as shown in Ref. [25].
[1] H. Darcy, Les Fontaines Publiques de la Ville de Dijon (Dalmont, Paris, 1856).

[2] J. Keller, Nonlinear Partial Differential Equations in Engineering and Applied Science, Proceedings of the Conference, Kingston, R.I., June 4-8, 1979 (Marcel Dekker, Inc., New York, 1980), pp. 429-443.

[3] E. Sanchez-Palencia, Nonhomogeneous Media and Vibration Theory (Springer-Verlag, Berilin, New York, 1980).

[4] U. Hornung, Homogenization and Porous Media (Springer, New York, 1997).

[5] W. Gray and K. O’Neill, Water Resour. Res. 12, 148 (1976).

[6] S. Whitaker, Transp. Porous Media 1, 3 (1986).

[7] J. Bear, Dynamics of Fluids in Porous Media (Elsevier, New York, 1988).

[8] M. Hassanizadeh, Transp. Porous Media 9, 207 (1986).

[9] M. Balhoff, A. Mikelić, and F. Wheeler, Transp. Porous Media 81, 35 (2010).

[10] M. Leverett, Trans. AIME 142, 152 (1941).

[11] J. Stokes, D. Weitz, J. Gollub, A. Dougherty, M. Robbins, P. Chaikin, and H. Lindsay, Phys. Rev. Lett. 57, 1718 (1986).

[12] D. G. Avraam and A. C. Payatakes, J. Fluid Mech. 293, 207 (1995).

[13] D. G. Avraam and A. C. Payatakes, Generalized Relative Permeability Coefficients During Steady-State Two-Phase Flow in Porous Media, and Correlation with the Flow Mechanisms, in Multiphase Flow in Porous Media (Springer, 1995), pp. 135-168.
[14] C. D. Tsakiroglou, D. G. Avraam, and A. C. Payatakes, Adv. Water Resour. 30, 1981 (2007).

[15] K. T. Tallakstad, G. Løvoll, H. A. Knudsen, T. Ramstad, E. G. Flekkøy, and K. J. Måløy, Phys. Rev. E 80, 036308 (2009).

[16] K. T. Tallakstad, H. A. Knudsen, T. Ramstad, G. Løvoll, K. Måløy, R. Toussaint, and E. G. Flekkøy, Phys. Rev. Lett. 102, 074502 (2009).

[17] X. Xu and X.-P. Wang, SIAM J. Appl. Math. 71, 1753 (2011).

[18] J. Joanny and M. Robbins, J. Chem. Phys 92, 32063212 (1990).

[19] E. Schaffer and P. Z. Wong, Phys. Rev. E 61, 5257 (2000).

[20] X.-P. Wang, T. Qian, and P. Sheng, J. Fluid Mech. 605, 59 (2008).

[21] W. Ren and W. E, Phys. Fluids 23, 072103 (2011).

[22] P. Sheng and M. Zhou, Phys. Rev. A 45, 5694 (1992).

[23] T. Qian, X.-P. Wang, and P. Sheng, Phys. Rev. E 68, 016306 (2003).

[24] T. Qian, X.-P. Wang, and P. Sheng, J. Fluid Mech. 564, 333 (2006).

[25] T. Qian, X.-P. Wang, and P. Sheng, Phys. Rev. Lett. 93, 094501 (2004).

[26] L. M. Hocking, J. Fluid Mech. 79, 209 (1977).

[27] M. Gao, Ph.D. thesis, Hong Kong University of Science and Technology, 2012.

[28] M. Gao and XP. Wang, J. Comput. Phys. 231, 1372 (2012).

[29] X.-P. Wang and Y.-G. Wang, Methods Appl. Anal. 14, 287 (2007). 